\title{
Role of freezing and fear in avoidance decrements following mammillothalamic tractotomy in cat: I. Two-way avoidance behavior'
}

\author{
E, E. KRIECKHAUS, POULTRY HUSBANDRY, UNIVERSITY OF CALIFORNIA AT DAVIS
}

CARL C. CHI, YALE UNIVERSITY

\begin{abstract}
In experiment 1 , six cats.were trained in a two-way conditioned avoidance response (CAR) and then received lesions aimed at the mammillothalamic tracts (MTT). Avoidance decrements following MTT lesions were not related to changes in freezing behavior. In experiment 2, four cats were trained in the CAR and then given intensive fear conditioning (pairing of buzzer and unavoidable foot shock). On subsequent CAR test sessions, avoidance latencies for trials on which the buzzer was added were significantly ( $\mathrm{p}<.01)$ shorter than on trials without the buzzer. Thus, a stimulus (the buzzer) capable of eliciting fear and freezing much stronger than that seen following MTT lesions actually facilitated the $\mathrm{CAR}$ (reduced avoidance latencies). This and the results of experiment I do not support the hypothes is that avoidance decrements following MTT lesions are attributable to increased fear or freezing.
\end{abstract}

Although it has been shown that in cats retention of a two-way (shuttle) conditioned avoidance response (CAR) is severely reduced following lesions of the mammillothalamic tracts (Thomas, Fry, Fry, Slotnick, \& Krieckhaus, 1963; Krieckhaus, 1964), the exact role of the mammillothalamic tract (MTT) in avoidance behavior is not clear. Thomas et al (1963) suggested that the avoidance decrements might be a result of MTT lesions increasing the likelihood that the innate response of freezing would become prepotent when fear producing stimuli were presented, while Krieckhaus (1964) proposed that the observed changes in freezing are more likely only secondary to an immediate effect on avoidance behavior. The present study will evaluate the role of freezing and other manifestations of fear in avoidance decrements following MTT lesions.

\section{EXPERIMENT 1}

In experiment 1 the intensity of responses such as freezing and immobility which are capable of competing with the CAR, was evaluated following MTT lesions. In earlier work (Thomas et al, 1963; Krieckhaus, 1964) the compartments of the avoidance apparatus were so small (floor area was 12 in. $x 18$ in.) that if the cat did not cross the hurdle there was little else it could do but remain motionless. This made it difficult to assess accurately whether the MTT lesions had really increased immobility and freezing. In the present experiment we repeated the prior work but used a larger avoidance apparatus.

\section{Method}

Six mongrel cats, four males and two females, were trained in the two-way CAR procedure described by Thomas et al (1963), except that in addition to the light CS a tone was added; also each compartment of the shuttle box was $45 \mathrm{in.}$ long, $24 \mathrm{in}$. wide and 40 in. high. When a cat had reached the learning criterion, electrolytic lesions were stereotaxically aimed bilaterally at MTT, Lesioning and histological procedures have been described by Thomas et al (1963). Postoperatively animals were tested for three days (60 trials) under preoperative conditions, except that no shock was administered. They were then retrained to criterion.

\section{Results}

In three cats the lesions missed the MTTs completely. On the 60 postoperative test trials cats made 60,60 and 59 responses and on retraining required 0,1 and 0 shocks to relearn. They also showed no changes in any manifestations of fear. Two cats with partial unilateral MTT transection made 14 and 40 responses and required 3 and 1 shocks, respectively, to relearn. One cat in which both MTTs were completely transected made no CAR postoperatively and required 12 shocks to relearn. (It had required 70 shocks to learn initially.) The above results are in accordance with our earlier reports concerning the effect of complete, partial and no lesions of the MTT on retention and relearning of a two-way CAR (Thomas et al,. 1963; Krieckhaus, 1964)。

Although the cat with complete bilateral MTT lesions showed decreased locomotion postoperatively, its behavior was similar to that of a normal, quiet cat, i.e., it made relaxed head movements and meowed, particularly when the CS came on, and yawned occasionally. The two cats with partial MTT lesions not only showed no marked increase in outward signs of fear such as immobility or freezing but usually exhibited behavior antagonistic to freezing. For the cat with the smaller, partial MTT lesions, on those trials on which it did not avoid, including the first such trial, when the CS came on the cat went up to the hurdle, stopped, turned its head and eyes rapidly as if confused, looked back over its shoulder, quickly moved away from the hurdle and returned to the back of the compartment; only then did the cat display any immobility or freezing. The cat with the larger, partial MTT lesion, with CS onset, manifested obvious autonomic signs of fear such as pupillary dilation and pilo-erection but was often observed to walk around the compartment with no particular orientation toward the door, or to walk up to the hurdle, place its front paws on it, look over into the other side, and then turn and walk away.

\section{EXPERIMENT 2}

Although these results offer little support for the hypothesis that the avoidance decrements following 
MTT lesions are attributable to increased freezing, it is still possible that an aspect of strong fear other than freezing or immobility might mediate these avoidance decrements. Indeed, there is evidence, discussed by Kenyon \& Krieckhaus (1965), that increased fear can produce decrements in the two-way CAR. The purpose of the present experiment is to ascertain whether a tremendous increase in fear could produce avoidance decrements comparable to those seen following MTT lesions. When each cat had reached the learning criterion, which previously (e.g., Thomas et al, and experiment 1) was followed by MTT lesioning, it was given intense fear conditioning to a buzzer. The effect of this conditioned fear on the CAR was then evaluated by comparing the number and the latencies of the CARs on trials with and without the buzzer.

\section{Method}

Four naive, mongrel cats were trained in a two-way CAR, Procedures and apparatus are described by Thomas et al (1963). When each cat reached criterion, it was given fear conditioning-six CS-US pairings (two per day for three days). The cat was placed in a box similar to the avoidance apparatus for $1 \mathrm{hr}$. After a randomly determined length of time (mean $=30 \mathrm{~min}$.) a CS, the attenuated sound of a door buzzer, was presented for 34 sec. The last 4 sec. of this CS overlapped the $4 \mathrm{sec}$. US $(4 \mathrm{ma}$ ). Following this fear conditioning, the cat was returned to the shuttle tox for 32 trials of avoidance testing ( 16 trials per day for two days). On the first and third blocks of four trials of each daily session the cat was tested as before the CS-US pairings i.e. with the light CS only; on the second and fourth blocks the buzzer was turned on simultaneously with the light CS. Following this testing the animals received 10 more CS-US pairings (two per day for five days). The procedure was the same except the pairings took place in alternate compartments of the avoidance apparatus and the US was $7 \mathrm{ma}$. Following two more days of avoidance testing. with and without the buzzer, the experiment was terminated.

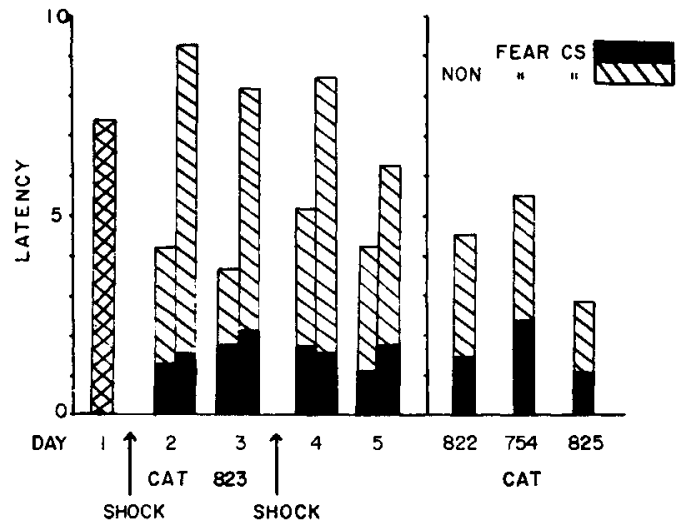

Fig. 1. Avoidance latencies with and without buzzer (fear CS) Striped columns represent average latency to cross the hurdle for blocks of 4 trials without buzzer, while solid columns are with the buzzer. Day 1 is the last day before CS-US pairings. The first column for each subsequent day represents the first block of 4 trials and the second column the second block. Days 2 and 3 are the 2 days following the first CS-US pairings and days 4 and 5 follow the second pairings. For the remaining 3 cats (right side of figure) each column represents total mean latencies for all trials with and without buzzer.

\section{Results}

By the third CS-US pairing the cats showed strong and intense freezing reactions to the buzzer. By the second pairing in the second series (in the shuttle box with $7 \mathrm{ma}$ shock), the cats exhibited intense fear reactions even when the buzzer was noton. When the buzzer did come on, fear was intensified to a degree that has rarely been observed in our laboratory in either normal or lesioned cats. The animals defecated and salivated profusely and alternated between increased freezing and an extremely rapid, stereotyped scratching at the walls.

In spite of these intense reactions during the CS-US pairings, in subsequent test sessions no cat failed to avoid on any of the trials on which the buzzer was presented. With buzzer onset, all cats showed a marked fear reaction which consisted of pilo-erection, pupillary dilation and an immediate crouching. A cat usually remained frozen in this manner for approximately 1 sec. and would then suddenly jump across the hurdle in one quick motion. Mean avoidance latencies of cat No. 823, which are typical of the other three cats, are depicted in the left portion of Fig. 1. For the other three cats, total avoidance latencies with and without the buzzer are presented in the right portion of Fig. 1. When latencies were analyzed separately for each cat for each of the four days of testing, mean latencies with buzzer were, in each case, significantly shorter than those without buzzer (p<.01; t-tests).

\section{Discussion}

Present results offer little support for the hypothesis that MTT lesions produce decrements in avoidance behavior by directly increasing fear or its manifestations such as freezing: When cats with partial MTT lesions in experiment 1 failed to avoid they often displayed behavior incompatible with freezing, and in experiment 2, introduction of a stimulus capable of eliciting extreme fear produced, with no exceptions, improvement, not decrements in the CAR. It is significant that the normal cats in experiment 2 clearly manifested more freezing both during the initial few seconds when the buzzer was turned on in avoidance testing, and during the CS-US pairings than the cat in experiment 1 following bilateral mammillothalamic tractotomy.

\section{References}

Kenyon, I., \& Krieckhaus, E. E. Decrements in one-way avoidance learning following septal lesions in rats. Psychon. Sci.. 1965 , 3, 113-114.

Krieckhaus, E. E. Decrements in avoidance behavior following mammiliothalamic tractotomy in cats. J. Neurophysiol., 1964, $27,753-767$.

Thomas, G. J., Fry, W. J., Fry, F. J., Slotnick, B. M., \& Krieckhaus, E. E. Behavioral effects of mammillothalamic tractotomy in cats. J. Neurophysiol., 1963, 26, 857-876.

\section{Note}

1. This research was supported by Research Grant 10885 from the National Science Foundation to the Biophysical Research Laboratory, University of Illinois and by Research Grant MY 647 from the National Institute of Mental Health to Yale University. 